\title{
An optimizing nested MIMO array with hole-free difference coarray
}

\author{
Liu Qiaoge ${ }^{1, a}$, Wang Buhong ${ }^{1}$, Li Xia $^{1}$,Tian Jiwei ${ }^{1}$, Cheng Tianhao ${ }^{1}$,Liu Shuaiqi ${ }^{2}$ \\ ${ }^{1}$ School of Information and Navigation, Air Force Engineering University, 710077, Xi'an ,Shaanxi ,China \\ ${ }^{2}$ People's Liberation Army 93995 troops, 710306, Xi'an ,Shaanxi ,China
}

\begin{abstract}
According to the newly proposed nested MIMO (Multiple-Input Multiple-Input Multiple Output Multiple Array) array design method, we propose to replace the traditional nested array into an optimizing nested array, ie, to optimizing nested MIMO array design. It not only retains the original advantage of nested MIMO array design closed expression with array element position and degree of freedom(DOF), but also greatly improves the array aperture and DOF. Optimizing nested MIMO array firstly uses the optimizing nested array as the transmitting and receiving arrays, and then make the difference set processing for the coarray of MIMO array (coarray, CA). By properly designing the array spacing of the transmitting and receiving arrays, we can obtain a non-porous difference array. When the total number of array elements is given, by analyzing the characteristics of the array structure, the best array element number of the transmitting and receiving arrays can be obtained. Simulation experiments show that compared with the nested MIMO array design, the proposed method can effectively expand the array aperture, increase the DOF, and increase the DOA estimation accuracy of the MIMO radar without increasing the number of actual array elements.
\end{abstract}

\section{Introduction}

MIMO radar[1-3] is a research hotspot in recent years. It can create a virtual array with larger aperture and higher DOF by transmitting orthogonal or incoherent waveforms and receiving echo signals at the receiving end using matched filters.

However, transmitting array and the receiving array of MIMO rader are all Uniform linear arrays (ULA), aperture length of the ULA is a little small, and the formed virtual array is only a combination of the transmitting array and the receiving array, so the virtual aperture of MIMO radar is limited leading to the degree of freedom is also limited.

In order to solve this problem, we can improve the DOF of MIMO radar by increasing the DOF of transmitting and receiving arrays. In the early research, the sparse array with closed expressions such as nested array[4]. coprime array[5] and minimum redundant array (MRA)[6] have been proposed. These arrays can provide larger apertures and higher DOF than ULA by utilizing second-order statistics of received data. Therefore, even when the number of sources is greater than the number of elements, they can effectively detect the location of all sources by DOA estimation.

In this regard, C.C. Weng proposed a minimum redundant MIMO array[7], which uses the minimum redundant array as the transmitting and receiving arrays, and performs the difference processing on sum coarray of the MIMO array to obtain the virtual extension of the

\footnotetext{
${ }^{\text {a }}$ Liu Qiaoge: 18829292513@163.com
}

number of receiving array elements. It can greatly improve the DOF of the array, but usually involves a large amount of computation. E. Boudaher proposed a coprime MIMO array[8], which replaced the minimum redundant array[7] with the coprime array. Although the calculation is reduced and the DOF of the array is improved, his difference array is a line array with hole, which leads to the inability to use the Spatial Smoothing (SS) algorithm to decorrelate the signal for accurate DOA estimation. M. Yang proposed a nested MIMO array design[9] with nested arrays as the transmitting and receiving arrays, and makes the difference set processing for the coarray of MIMO array, thus obtaining the virtual extension of the number of receiving array elements. Although it can further improve the DOF of MIMO radar and obtain a uniform line array without holes, since the DOF of nested array is limited, the DOF of nested MIMO array is also limited.

To overcome these shortcomings, the paper proposes an optimizing nested MIMO array.The main improvement is to replace the nested array of the transmitting end and the receiving end to the optimizing nested array[10]. The optimizing nested array is proposed aiming to overcome the existing shortcomings of the nested array. The disadvantage of the nested array is that when it is nested in two layers. The difference array is a uniform linear array without hole. However, it's DOF is limited. when it is nested in multiple layers,although it can provide higher DOF, it's difference array is not a uniform line array without hole[4]. The optimizing nested 
array proposed in this paper contains all the advantages of nested array while providing a higher DOF. The position of the array element, the length of the array aperture and the DOF of the difference array have a closed expression, and the difference array is a linear array with larger aperture length and higher DOF meanwhile without hole.

the Optimizing nested MIMO array firstly uses an optimizing nested array as the transmitting array of the MIMO radar, and secondly we calculate the number of consecutive elements of the difference array of transmitting array, that is, the aperture length of the transmitting array, and finally we use another optimizing nested arrays with a larger array element spacing $\mathrm{S}$ as receiving array.

By reasonably designing $S$, we can obtain a difference array without hole with larger aperture length and a higher DOF. Although nested MIMO arrays[9] can also obtain difference array without hole, optimizing nested MIMO array has larger aperture length and higher DOF for better DOA estimation performance.

\section{Optimizing nested array structure}

The optimizing nested array is composed of two uniform linear arrays and a single element. The first level uniform linear array is composed of $M_{1}$ elements with elements spaced by $\mathrm{d}$, and the second level uniform linear array is composed of $M_{2}$ elements with elements spaced by $\left(M_{1}+2\right) d$.The individual element is placed at a distance $\left(M_{1}+1\right) d$ from the second level uniform linear array, as shown in figure1. Therefore, the total number of elements is equal to $M_{1}+M_{2}+1$.

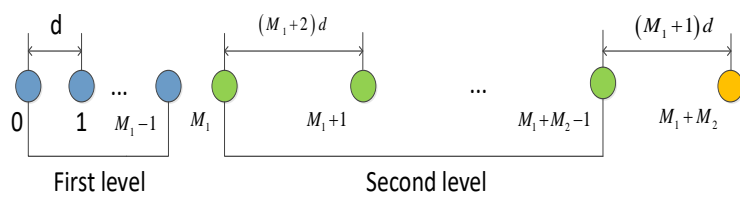

Figure 1. Optimizing nested array structure

From Figure 1, we can get the aperture length of the optimizing nested array.

$$
\begin{aligned}
& L=\left[M_{1}+\left(M_{2}-1\right)\left(M_{1}+2\right)+M_{1}+1\right] d \\
& ==\left(M_{1} M_{2}+2 M_{2}+M_{1}-1\right) d
\end{aligned}
$$

According to the symmetry characteristic of the difference array, the DOF of the optimizing nested array is

$$
D_{M}=2 \frac{L}{d}+1=2 M_{1} M_{2}+4 M_{2}+2 M_{1}-1
$$

According to equation (2), we can optimize the number of array elements of the two level uniform linear array with the total number of array elements fixed to $M=M_{1}+M_{2}+1$, so that the array has the highest DOF, and the optimization problem about total number of array elements can be solved by arithmetic - Geometric mean inequality. Table1 summarizes the optimal allocation problem for the array elements.

Table1. the optimal allocation of array elements in a optimizing nested array

\begin{tabular}{|c|c|}
\hline$M$ & Optimal value of $M_{1}, M_{2}$ \\
\hline Even & $M_{1}=\frac{M}{2}-1, \mathrm{M}_{2}=\frac{M}{2}$ \\
\hline Odd & $M_{1}=\frac{M-1}{2}-1, \mathrm{M}_{2}=\frac{M+1}{2}$ \\
\hline
\end{tabular}

Substitute the best value of $M_{1}, M_{2}$ into equation (2), we can get the DOF of the optimizing nested array

$$
D_{M}=\left\{\begin{array}{l}
\frac{M^{2}}{2}+2 M-3, M \text { is even } \\
\frac{M^{2}}{2}+2 M-\frac{7}{2}, M \text { is odd }
\end{array}\right.
$$

\section{MIMO Radar signal model}

Suppose there is a colocated MIMO radar system with M transmitting antennas and $\mathrm{N}$ receiving antennas. Both the transmitting and receiving arrays are all optimizing nested arrays. Their position sets are represented as $\left\{p_{T, m}\right\}_{m=1}^{M}$ and $\left\{p_{R, n}\right\}_{n=1}^{N},\left\{p_{T, m}\right\}$ and $\left\{p_{R, n}\right\}$ are integer times of half-wavelength. The transmitting array transmits $\mathrm{M}$ mutually orthogonal waveforms, and the complex envelope of the signal transmitted by the $\mathrm{mth}$ transmitting element is

$$
s_{m}(t)=\sqrt{\frac{E}{M}} \phi_{m}(t), m=1, \ldots, M
$$

Where $\mathrm{t}$ is the time parameter within a radar pulse, and $\mathrm{E}$ is the total transmitting energy within a radar pulse, $\phi_{m}(t)$ is the mth base-lobe waveform signal. Since the waveforms transmitted by the different antennas are orthogonal, the waveform is normalized to have unit energy, i.e. $\int_{T}\left|\phi_{m}(t)\right|^{2} d t=1, m=1, \ldots, M$, where $\mathrm{T}$ is the radar pulse width.

Assuming there are $\mathrm{Q}$ sources, the receiving complex vector of the receiving array observation value can be written as

$$
\mathbf{x}(t, \tau)=\sum_{q=1}^{Q} r_{q}(t, \tau) \mathbf{b}\left(\theta_{q}\right)+\mathbf{n}(t, \tau)
$$

$\tau$ is the number of pulse, $\mathbf{b}(\theta)=\left[e^{j k p_{R, 1} \sin \theta}, e^{j k p_{R, 2} \sin \theta}, \ldots, e^{j k p_{R, N} \sin \theta}\right]^{T}$ is the reception steering vector, where $k=\frac{2 \Pi}{\lambda}$ and $\mathbf{n}(t, \tau)$ is the $N \times 1$ dimensional zero-mean Gaussian white noise, $r_{q}(t, \tau)=\sqrt{\frac{E}{M}} \beta_{q}(\tau) \mathbf{a}^{T}\left(\theta_{q}\right) \phi(t)$ is the radar echo about 
the qth source. For this formula, $\beta_{q}(\tau)$ and $\theta_{q}$ respectively are the reflection coefficient and the spatial angle, $\mathbf{a}\left(\theta_{q}\right)=\left[e^{j k p_{T, 1} \sin \theta_{q}}, e^{j k p_{T, 2} \sin \theta_{q}}, \ldots, e^{j k p_{T, M} \sin \theta_{q}}\right]^{T}$ is the transmitting steering vector of the qth source, and the symbol $(\cdot)^{T} \quad$ indicates the transposition, $\phi(t) \triangleq\left[\phi_{1}(t), \ldots, \phi_{M}(t)\right]^{T}$ is the waveform vector. It is assumed that the reflection coefficient of each source is constant throughout the pulse period, but varies betwwen different pulse periods.

Using the orthogonal characteristics of the transmitting waveform, a matched filter can be used to extract the $N \times 1$ dimensional received data of the $m$ th waveform in equation (5), as shown below.

$$
\boldsymbol{x}_{m}(\tau) \triangleq \int_{T} \boldsymbol{x}(t, \tau) \phi_{m}^{*}(t) d t, m=1, \ldots, M
$$

$(\cdot)^{*}$ is the conjugate operator. Superimpose $\boldsymbol{x}_{1}(\tau), \ldots, \boldsymbol{x}_{M}(\tau)$ in a column vector, we can get the virtual data vector of the $M N \times 1$ dimension, as shown below

$$
\begin{aligned}
\boldsymbol{y}_{\text {MIMO }}(\tau) & \triangleq\left[\boldsymbol{x}_{1}^{T}(\tau), \ldots, \boldsymbol{x}_{M}^{T}(\tau)\right]^{T} \\
& =\sqrt{\frac{E}{M}} \sum_{q=1}^{Q} \beta_{q}(\tau) \mathbf{a}\left(\theta_{q}\right) \otimes \mathbf{b}\left(\theta_{q}\right)+\hat{\mathbf{n}}(\tau)
\end{aligned}
$$

$\otimes$ represents the Kronecker product.

$$
\begin{aligned}
& \mathbf{U}_{\text {MIMO }}(\theta) \triangleq \mathbf{a}(\theta) \otimes \mathbf{b}(\theta) \\
& =\left(e^{j k\left(p_{T, 1}+p_{R, 1}\right) \sin \theta}, e^{j k\left(p_{T, 1}+p_{R, 2}\right) \sin \theta}, \ldots, e^{j k\left(p_{T, 1}+p_{N}\right) \sin \theta},\right. \\
& \left.\ldots, e^{j k\left(p_{T, M}+p_{R, 1}\right) \sin \theta}, e^{j k\left(p_{T, M}+p_{R, 2}\right) \sin \theta}, \ldots, e^{j k\left(p_{T, M}+p_{N}\right) \sin \theta}\right)^{T}
\end{aligned}
$$

is the transpose vector of the $M N \times 1$ dimensional virtual array and $\hat{\mathbf{n}}(\tau)$ is the noise item of the $M N \times 1$ dimension.

It can be seen from the above analysis that MIMO radar transmits mutually orthogonal waveforms from the transmitting end and receives waveforms by the matched filter bank at the receiving end, so that it can synthesize a virtual array with $\mathrm{MN}$ elements, and the array position set is expressed as

$$
\left\{p_{g}\right\}=\left\{p_{T, m}+p_{R, n} \mid m=1,2, \ldots, M ; n=1,2, \ldots, N\right\}
$$

$g=1,2, \ldots, M N$, from equation (9), we can see that the array element position of the MIMO radar virtual array is the direct sum of the position of the transmitting array and the receiving array element. Therefore, we call this virtual array as a coarray of MIMO radar.

\section{Optimizing nested MIMO array}

\subsection{Array model}

Optimizing nested MIMO array can be built in three steps
1) Use an optimizing nested array with $M$ elements as the transmitting array, it's element position is expressed as

$$
\left\{p_{T, m}\right\}=\left\{a_{m} \mid m=1,2, \ldots, M\right\}
$$

$\left\{a_{m}\right\}_{m=1}^{M}$ is the set of element position of the optimizing nested array.

2) The receiving array is another optimizing nested array with a larger element spacing $\mathrm{S}$, and it's element position is expressed as

$$
\left\{p_{R, n}\right\}=\left\{b_{n} \cdot S \mid n=1,2, \ldots, N\right\}
$$

$\left\{b_{n}\right\}_{n=1}^{N}$ is the sequence of the optimizing nested array element position. Substitute equations (10) and (11) into equation (9), the position set of the MIMO radar coarray can be obtained.

$$
\begin{gathered}
\left\{p_{T, m}+p_{R, n}\right\}=\left\{a_{m}+b_{n} \cdot S \mid m=1,2, \ldots, M ;\right. \\
n=1,2, \ldots, N\}
\end{gathered}
$$

3 ) Gain the difference array of the MIMO radar coarray, that is, the position set of the difference coarray

$$
\begin{aligned}
& \left\{p_{T, m}+p_{R, n}-p_{T, m^{\prime}}-p_{R, n^{\prime}}\right\} \\
= & \left\{a_{m}+b_{n} \cdot S-a_{m^{\prime}}-b_{n^{\prime}} \cdot S \mid\right. \\
& \left.m, m^{\prime}=1,2, \ldots, M ; n, n^{\prime}=1,2, \ldots, N\right\}
\end{aligned}
$$

The virtual transposition vector of difference coarray obtained by the second part is

$$
\begin{array}{r}
\mathbf{U}_{D C}(\theta)=\left(e^{j k\left(a_{1}+b_{1} \cdot S-a_{1}-b_{1} \cdot S\right) \sin \theta}, \ldots,\right. \\
\left.e^{j k\left(a_{M}+b_{N} \cdot S-a_{M}-b_{N} \cdot S\right) \sin \theta}\right)^{T}
\end{array}
$$

The position set of the difference coarray can be expressed as

$$
\begin{aligned}
&\left\{p_{T, m}+p_{R, n}-p_{T, m^{\prime}}-p_{R, n^{\prime}}\right\} \\
&=\left\{a_{m}-a_{m^{\prime}}+\left(b_{n}-b_{n^{\prime}}\right) \cdot S \mid m, m^{\prime}=1,2, \ldots, M\right. \\
& n, n^{\prime}=1,2, \ldots, N \\
&\left(a_{m}-a_{m^{\prime}}\right) \text { and }\left(b_{n}-b_{n^{\prime}}\right) \text { are respectively }
\end{aligned}
$$

difference arrays of the transmitting and receiving arrays. Under this structural designing, As long as $\mathrm{S}$ is equal to $D_{M}$, we can find that the difference coarray of MIMO radar is a line array without hole[11]. In addition, since the transmitting and receiving arrays have closed expressions, the difference coarray of the optimizing nested MIMO array also has closed expression.

Figure 2 shows an example of an optimizing nested MIMO array with a five-element transmitting array and a four-element receiving array. Since the difference coarray has symmetry characteristic, only the non-negative part of the difference coarray is shown in the figure2 [4]. Since ODF of the difference array of the transmitting array is 19 , there is $S=19$ in the receiving array. From Figure 2, we can see the aperture length of the optimizing nested MIMO array with five transmitting elements and four receiving elements $L=123$, and the difference coarray is a uniform linear array without hole. 


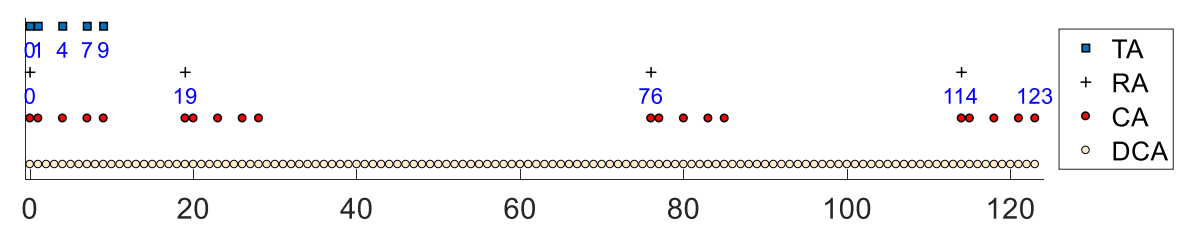

Figure 2. An example of the optimizing nested MIMO with five-element transmitting array and four-element receiving array

\subsection{Comparison of DOF between optimizing nested MIMO array and nested MIMO array}

The variation about the DOF of the two arrays structure with respect to the total number of element $G$ is listed in Table 2. It can be clearly seen that the DOF of the two arrays structure has increased significantly with the increase of the $\mathrm{G}$ value, but the DOF of the optimizing nested MIMO has increased more than the DOF of the nested MIMO array.

Tab 2 . Comparison of the Degree of Freedom of Nested MIMO Arrays and Nested MIMO Arrays with Regard to the Total Number of Array Elements G

\begin{tabular}{|c|c|c|c|c|c|c|}
\hline $\mathrm{G}$ & 7 & 9 & 14 & 19 & 22 & 25 \\
\hline$M_{N}$ & 3 & 4 & 7 & 9 & 11 & 12 \\
\hline$N_{N}$ & 4 & 5 & 7 & 10 & 11 & 13 \\
\hline $\begin{array}{l}\text { DOF of } \\
\text { nested } \\
\text { MIMO }\end{array}$ & 77 & 187 & 961 & 2891 & 5041 & 8051 \\
\hline $\begin{array}{l}\text { DOF of } \\
\text { Optimizing } \\
\text { nested } \\
\text { MIMO }\end{array}$ & 91 & 247 & 1225 & 3685 & 6241 & 9951 \\
\hline
\end{tabular}

\section{Computer simulation experiment}

In this part, we compare the performance of optimizing nested MIMO and nested MIMO by computer simulation, which proves the superiority of optimizing nested MIMO. Experiment 1: DOA Estimated Success Rate

First, we compare the DOA estimation success rate of the two arrays structure. The DOA estimation method is the Spatial Smoothing-based MUSIC algorithm[4]. We take the example of an optimizing nested MIMO array and a nested MIMO array with a total number of array elements of 9, the coarray and the difference coarray of optimizing nested MIMO array are shown in Figure 2. Assuming that the number of snapshots is 1500, Figures 3 show the normalized MUSIC spectrum in this case when 60 uncorrelated sources with the same energy are uniformly distributed in the spatial frequency of -1 to 1 . The normalized MUSIC spectrumes show that both the optimizing nested MIMO array and the nested MIMO array can correctly estimate the number of sources far beyond the total number of elements.

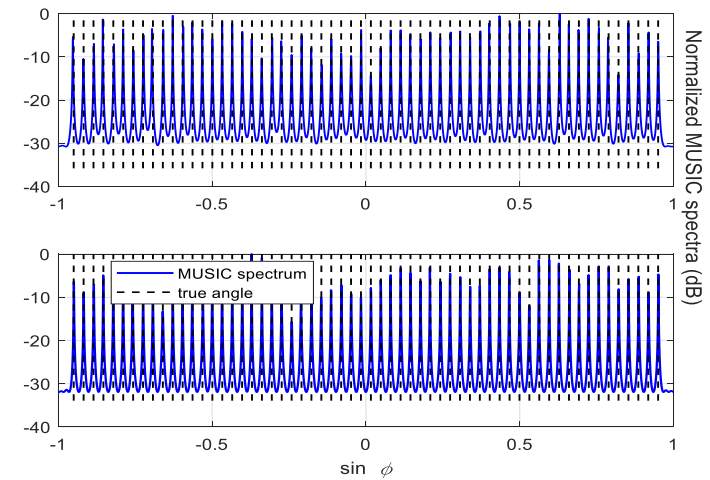

Fig 3. The normalized MUSIC spectrum with a total number of sources of 60 . The vertical dashed line is the true location of the

source. The figure above shows the normalized MUSIC spectrum of the nested MIMO array. The figure below shows the normalized MUSIC spectrum of the optiming nested MIMO array

However, when the total number of sources increases to 70, his normalized MUSIC spectrum is shown in Figure 4. It can be seen that the optimizing nested MIMO array can still accurately estimate the position of all sources, but the nested MIMO array failed. It can be infered that the number of sources that can be estimated by optimizing nested MIMO array is larger than the number of sources that can be estimated by the nested MIMO array.

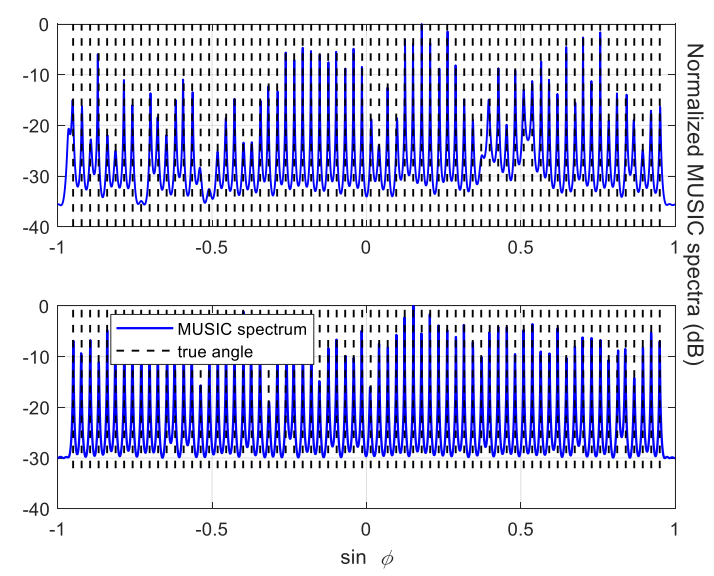

Fig 4. The normalized MUSIC spectrum with a total number of sources of 70. The vertical dashed line is the true location of the source. The figure above shows the normalized MUSIC spectrum of the nested MIMO array. The figure below shows the normalized MUSIC spectrum of the optimizing nested MIMO array

Experiment 2: Comparison of DOA Estimation Accuracy

In order to verify that the optimizing nested MIMO array has higher DOA estimation accuracy than the 
nested MIMO array, we make the optimizing nested MIMO array and the nested MIMO array with the total number of array elements of 9 as an example, assuming that there are 7 narrow-lobe uncorrelated sources respectively located in the $\left[-40^{\circ},-30^{\circ},-20^{\circ}, 0^{\circ}, 30^{\circ}, 40^{\circ}, 45^{\circ}\right]$ orientation. The root mean square error (RMSE) of the DOA estimate was calculated using Monte Carlo simulation, with the Monte Carlo simulation number set to 100 . The RMSE can be calculated by the following formula

$$
R M S E=\sqrt{\frac{1}{V Q} \sum_{v=1}^{V} \sum_{q=1}^{Q}\left(\hat{\theta}_{v, q}-\theta_{q}\right)^{2}}
$$

$\hat{\theta}_{v, q}$ represents the DOA estimate of the qth source in the vth Monte Carlo simulation experiment, and V represents the number of Monte Carlo simulations.

Figure 5 is the relationship between the estimated RMSE and the signal to noise ratio(SNR) in the above case. It can be seen from the figure that the estimated RMSE of the two arrays decreases with the increase of SNR, but under the same SNR, the estimated RMSE of the optimizing nested MIMO array is significantly lower than that of the nested MIMO array. The RMSE indicates that the DOA estimation accuracy of the optimizing nested MIMO array is higher than that of the nested MIMO array.

In summary, the performance of the optimizing nested MIMO array is better than the nested MIMO array. This is because the optimizing nested MIMO array has larger virtual aperture length and DOF when the total number of array elements is the same.

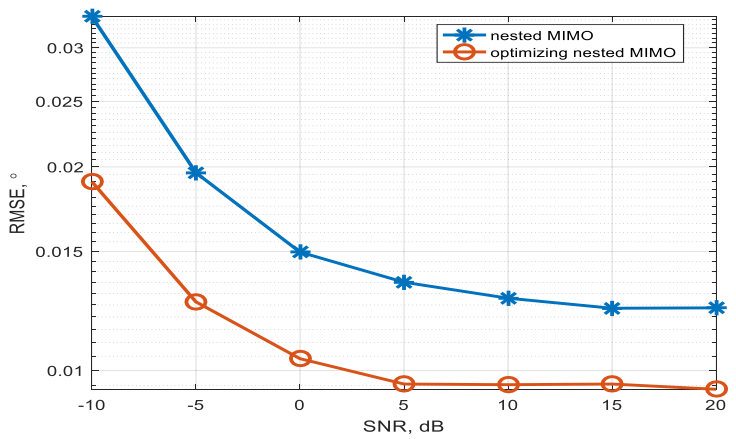

Fig 5. Signal-to-noise ratio and estimated root mean square error of two arrays with a total of 9 elements

\section{Conclusion}

In this paper, a new array design-the optimizing nested MIMO array design is proposed. It can be seen from the analysis and simulation of this paper that it not only has the closed expression of array element position and DOF, but also compared with the nested MIMO array,the array further expands the virtual array aperture, increases the DOF, improves the DOA estimation accuracy and the number of source that can be estimated, and the difference coarray of the optimizing nested MIMO array is a uniform line array without hole. This array structure is simple and easy to be obtained. It has increased the
DOF so that the DOA estimation performance has increased. This also has certain practical significance to research on radar DOA estimation. The proposed optimizing nested MIMO array overcomes the disadvantages of the existing minimum redundant MIMO array, coprime MIMO and nested MIMO array. Maximize the DOF under the premise that the amount of computation is small and the difference coarray is nonporous. However, this paper only improves the nested array of the transmitting and receiving ends. so that the DOF of the array is improved. As for whether there is a better overall array structure improvement scheme, further research is needed.

\section{References}

1. A. Hassanien, S.A. Vorobyov. Transmit energy focusing for DOA estimation in MIMO radar with colocated antennas. IEEE Transactions on Signal Processing, 59(6): 2669-2682(2011)

2. R. Amiri, F. Behnia, M.A.M Sadr. Efficient positioning in MIMO radars with widely separated antennas. IEEE Communications Letters, 21(7): 1569-1572(2017)

3. Y Wang, G. Huang, W. Li, et al. Colocated MIMO radar waveform-design based on two-step optimizations in spatial and spectral domains. Frontiers of Information Technology \& Electronic Engineering, 18(7): 1021-1032(2017)

4. P. Pal, P.P. Vaidyanathan. Nested arrays: A novel approach to array processing with enhanced degrees of freedom. IEEE Transactions on Signal Processing, 58(8): 4167-4181(2010)

5. Z. Shi, C. Zhou, Y. Gu, et al. Source estimation using coprime array: A sparse reconstruction perspective. IEEE Sensors Journal,17(3): 755765(2017)

6. J. Dong, R. Shi, Y. Guo. Minimum redundancy MIMO array synthesis with a hybrid method based on cyclic difference sets and ACO. International Journal of Microwave and Wireless Technologies, 9(1): 35-43(2017)

7. C.C. Weng, P.P. Vaidyanathan. Nonuniform sparse array design for active sensing.ASILOMAR, IEEE, 1062-1066(2011)

8. E. Boudaher, F. Ahmad, M.G. Amin. Sparsity-based direction finding of coherent and uncorrelated targets using active nonuniform arrays. IEEE Signal Processing Letters, 22(10): 1628-1632(2015)

9. M. Yang, L. Sun, X. Yuan, et al. A New Nested MIMO Array With Increased Degrees of Freedom and Hole-Free Difference Coarray. IEEE Signal Processing Letters, 25(1): 40-44(2018)

10. M. Yang, L. Sun, X. Yuan, et al. Improved nested array with hole-free DCA and more degrees of freedom. Electronics Letters, 52(25): 20682070(2016)

11. S. Qin, Y.D. Zhang, M.G. Amin. DOA estimation of mixed coherent and uncorrelated signals exploiting a nested MIMO system//Microwave and Antenna Subsystems for Radar, Telecommunications, and 
Biomedical Applications (BenMAS), 2014 IEEE

Benjamin Franklin Symposium on. IEEE, 1-3(2014) 